\title{
BMJ Open Treatment of multiple brain metastases using gadolinium nanoparticles and radiotherapy: NANO-RAD, a phase I study protocol
} Camille Verry, ${ }^{1,2}$ Lucie Sancey, ${ }^{3}$ Sandrine Dufort, ${ }^{4}$ Geraldine Le Duc, ${ }^{4}$
Christophe Mendoza, ${ }^{5}$ François Lux, ${ }^{3}$ Sylvie Grand, ${ }^{6}$ Josiane Arnaud, ${ }^{7}$
Jean Louis Quesada, ${ }^{5}$ Julie Villa, ${ }^{1}$ Olivier Tillement, ${ }^{3}$ Jacques Balosso ${ }^{1,2}$

To cite: Verry C, Sancey L, Dufort S, et al. Treatment of multiple brain metastases using gadolinium nanoparticles and radiotherapy: NANO$\mathrm{RAD}$, a phase I study protocol. BMJ Open 2019;9:e023591. doi:10.1136/ bmjopen-2018-023591

- Prepublication history for this paper is available online. To view these files, please visit the journal online (http://dx.doi. org/10.1136/bmjopen-2018023591).

Received 27 April 2018 Revised 10 August 2018 Accepted 27 September 2018

Check for updates

(C) Author(s) (or their employer(s)) 2019. Re-use permitted under CC BY-NC. No commercial re-use. See rights and permissions. Published by BMJ.

For numbered affiliations see end of article.

Correspondence to Dr Camille Verry; cverry@chu-grenoble.fr

\section{ABSTRACT}

Introduction Occurrence of multiple brain metastases is a critical evolution of many cancers with significant neurological and overall survival consequences, despite new targeted therapy and standard whole brain radiotherapy (WBRT). A gadolinium-based nanoparticle, AGuIX, has recently demonstrated its effectiveness as theranostic and radiosensitiser agent in preclinical studies. The favourable toxicity profile in animals and its administration as a simple intravenous injection has motivated its use in patients with this first in human study. Methods and analysis The NANO-RAD study is a phase I, first in human injection, monocentric, open-label, doseescalation study to investigate the safety, the tolerability and the spectrum of side effects of AGulX in combination with WBRT (30 Gy, 10 fractions of $3 \mathrm{~Gy}$ ) for patients with multiple brain metastases. Five dose escalation cohorts are planned: $15,30,50,75$ and $100 \mathrm{mg} / \mathrm{kg}$. A total of 15-18 patients will be recruited into this trial. The primary objective is to determine the maximum-tolerated dose of AGuIX nanoparticles combined with WBRT for the treatment of multiple brain metastases. Toxicity will be assessed using the National Cancer Institute Common Toxicity Criteria V.4.03. Secondary objectives are pharmacokinetic profile, distribution of AGuIX in metastases and surrounding healthy tissue visualised by MRI, intracranial progression-free survival and overall survival. Intracranial response will be determined according to Response Evaluation Criteria in Solid Tumour Criteria V.1.1 comparing MRI performed prior to treatment and at each follow-up visits.

Ethics and dissemination Approval was obtained from the ethics committee Sud Est V, France (Reference number 15-CHUG-48). The study was approved by the French National Agency for the Safety of Medicines and Health Products (ANSM) (Reference number 151519A-12). The results will be published in peer-reviewed journals or disseminated through national and international conferences.

Trial registration number NCT02820454; Pre-results.

\section{INTRODUCTION}

The occurrence of multiple brain metastases is a critical point of the evolution of many
Strengths and limitations of this study

- Phase I trial of first in man intravenous injection of theranostic gadolinium-based nanoparticles.

- One of the rare clinical trial proposed for improvement of the treatment of multiple brain metastases.

- Study with close follow-up of the patients in terms of clinical evaluation, imaging and pharmacokinetic. Monocentric study with few patients.

cancers with devastating neurological consequences and a very short overall survival of about 2 months without treatment ${ }^{1}$ and about 4.5 months with whole brain radiation therapy. ${ }^{23}$ This is a frequent situation in oncology since $25 \%$ of cancer patients have brain metastases in autopsy series. ${ }^{4}$ The three most commonly involved cancers are lung, breast and melanoma.

The current treatments' choice for brain metastases is based on their characteristics (number, size and location), the type of primary tumour and the performance status of the patient.

The main treatments are surgery, radiation therapy and systemic therapies (chemotherapy, hormonotherapy, targeted therapies and immunotherapy). Surgery is discussed for patients with a controlled primary disease and in the presence of a single brain metastasis. In such case, the 1-year intracranial control rate is approximately $50 \% .^{5}$ Adjuvant encephalic radiation is usually performed to increase local control. Stereotactic radiotherapy can deliver high doses per fraction with high accuracy. This dose escalation provides good results in terms of local control up to $80 \%$ at 1 year. ${ }^{1}$ This treatment is usually performed for patients having few metastases, usually less than three metastases, with a diameter less than $3 \mathrm{~cm}$ and no evolutive extracranial 
disease. Chemotherapy demonstrates a modest efficacy in brain metastases, probably related to a poor intracerebral distribution due to the blood-brain barrier. Similarly, targeted therapies (tyrosine kinase inhibitors, immunotherapies) are also of low efficacy in terms of response rate and overall survival for patients with multiple brain metastases. In addition, most studies evaluating these new therapies exclude patients with multiple brain metastases due to poor prognosis or fear of severe toxicity.

In the other situations, the reference treatment is whole brain radiotherapy (WBRT), used as a palliative treatment. The most commonly prescribed dose is $30 \mathrm{~Gy}$ in 10 fractions of $3 \mathrm{~Gy}$. The association of WBRT and chemotherapy did not yet demonstrate superiority despite interesting results with temozolomide in Phase II trials. ${ }^{6}$

In the last decades, numerous chemical radiosensitisers have been studied to improve efficacy of the radiation exposure. Among them, efaproxiral increases the offloading of oxygen into the tumour tissues and showed, after injection of $100 \mathrm{mg} / \mathrm{kg}$ half an hour before each radiotherapy session, significant results in terms of local response in a phase II study ${ }^{7}$ and a trend of improved overall survival in a phase III randomised trial including 515 patients (5.4 months vs 4.4 months). ${ }^{8}$ Conversely, motexafin gadolinium (possibly by triggering the depletion in intracellular antioxidants) injected at $5 \mathrm{mg}$ / $\mathrm{kg}$ 2-5hours before each session failed to increase the overall survival in a randomised trial of 401 young patients. However, the results highlighted an improvement of cognitive function and neurological symptoms. ${ }^{9}$

More recently, nanoparticles appeared as a new generation of radiosensitisers. These nanoparticles are based on elements like gold $(\mathrm{Z}=79),{ }^{10}$ hafnium $(\mathrm{Z}=72)^{11}$ or gadolinium $(Z=64)^{12}$ that display a high atomic number enabling greater interaction with the applied irradiation. Currently, none of them are used in clinic for radiosensitisation after intravenous administration, except the gadolinium-based nanoparticles, AGuIX, described in this clinical trial.

\section{The radiosensitising agent: AGuIX}

A new gadolinium-based nanoparticle agent, named AGuIX (Activation and Guidance of Irradiation by X-Ray), has recently demonstrated effectiveness as a radiosensitiser in vitro ${ }^{13-17}$ and in vivo. ${ }^{13-22}$ It is a theranostic drug: acting as MRI contrast agent enhancing tumours' contrast on $\mathrm{T}_{1}$-weighted images, ${ }^{23}$ as well as cytotoxic agent when combined with radiotherapy. ${ }^{17}$

These nanoparticles are composed of a polysiloxane core surrounded by cyclic ligands of gadolinium, derived from DOTA (1,4,7,10-tetraazacyclododecane-1,4,7,10-tetraacetic acid) and covalently grafted onto a polysiloxane matrix. The diameter is of $3 \pm 1.5 \mathrm{~nm}$ and the mass is $10 \pm 5 \mathrm{kDa}$ (figure 1). A simple intravenous administration allows the accumulation of the medication in the tumours by enhanced permeability and retention effect. ${ }^{24}$ The small size of the nanodrug $(<5 \mathrm{~nm})$ facilitates its renal clearance,$^{25}$ while keeping a blood half-life of 2 hours in

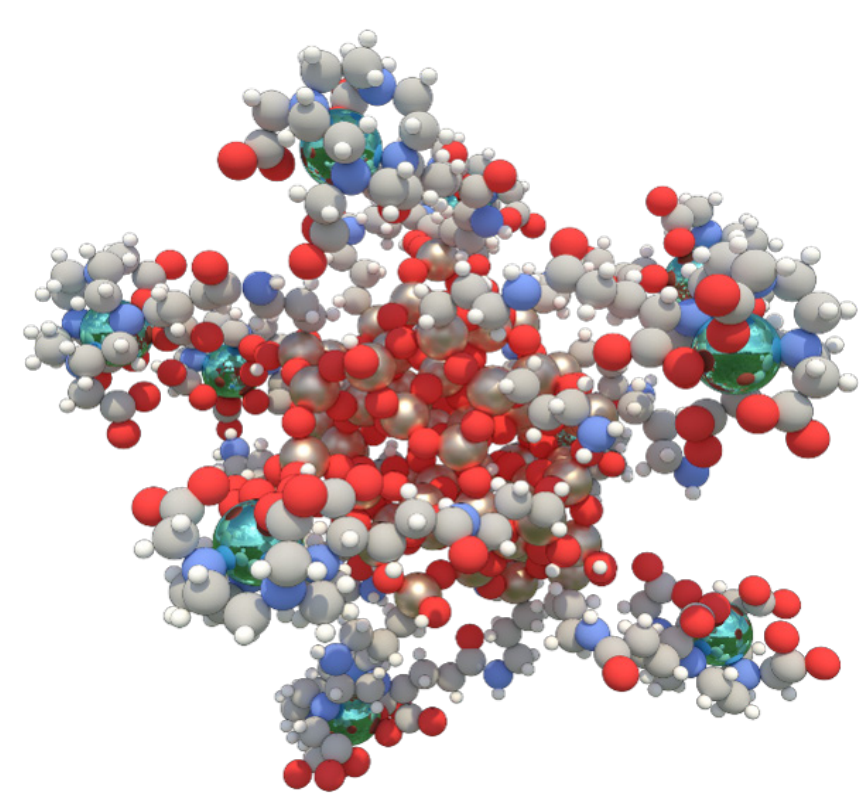

Figure 1 Schematic representation of AGulX. Gadolinium ions are chelated by 1,4,7,10-tetraazacyclododecane1,4,7,10-tetraacetic acid derivatives. Polysiloxane core ( $\mathrm{Si}$, metallic grey; $\mathrm{O}$, red; $\mathrm{H}$, white; $\mathrm{C}$, grey; $\mathrm{N}$, blue) is surrounded by covalently grafted chelates of gadolinium $(\mathrm{Gd}$, metallic green).

rats and 2.5 hours in monkeys, respectively. ${ }^{22}{ }^{26}$ Moreover, due to its rich gadolinium content, AGuIX leads to a high absorption of the photons delivered by the radiotherapy beam and/or secondary ionising species, which generates an enhanced local dose deposit yielding an enhanced creation of free radicals.

\section{In vitro experiments}

The addition of AGuIX (from $0.1 \mathrm{mM}$ to $1.0 \mathrm{mM}$ ) increases the irradiation effectiveness by a factor of 1.1-2.5 depending on the energy of the photon beam used (from $6 \mathrm{MV}$ to $50 \mathrm{kV}$, respectively) and the cell line studied. Such efficacy has been reported for head and neck squamous cell carcinoma SQ20B, ${ }^{21}$ glioblastoma U-87 MG,${ }^{15}$ cervical carcinoma HeLa, ${ }^{14}$ pancreatic adenocarcinoma Capan- $1{ }^{18}$ and melanoma B16F10 ${ }^{13}$ Interestingly, the radiosensitising effect is also occurring with heavy ion therapy. ${ }^{16}$

\section{In vivo experiments}

The efficacy of AGuIX as a radiosensitising agent has been demonstrated in six animal tumour models. An injection 24 hours before an irradiation of $20 \mathrm{~Gy}$ in a single session by synchrotron microbeam radiation therapy doubled the overall survival compared with the radiotherapy alone arm for a rat model of intracranial glioma $(9 \mathrm{~L}){ }^{20}$ This radiosensitising effect was confirmed on the same rat model using a conventional radiotherapy linear accelerator with a $20 \mathrm{~Gy}$ WBRT in 2 fractions of $6 \mathrm{MV}$ photons. ${ }^{22}$ The MRI analysis showed a strong enhancement of the tumour signal up to 24 hours with a very favourable 
tumour versus brain ratio. Moreover, the mean tumour volume measured 7 days after the irradiation with AGuIX was of $29 \mathrm{~mm}^{3}$ compared with $48 \mathrm{~mm}^{3}$ in the radiotherapy alone arm. Another model of WBRT after AGuIX injection has been implemented with mice bearing multiple brain metastases of melanoma (B16F10). ${ }^{13}$ A single fraction of 7 Gy was performed 3.5 hours after the injection with a significant increase of $25 \%$ of survival in the AGuIX combined with WBRT arm. Finally, a subcutaneous model of pancreatic tumour (Capan-1) has been implemented for $6 \mathrm{MV}$ photons irradiation. ${ }^{18} \mathrm{~A}$ single fraction of $10 \mathrm{~Gy}$ performed $15 \mathrm{~min}$ after injection induced a significant reduction of $50 \%$ of tumour growth, and a significant ILS of $56 \%$, compared with radiation alone.

\section{Animal toxicity studies}

Two studies have shown safety in mice, even at high doses, as well as a precise description of the renal elimination..$^{2527}$ In parallel, studies required by the regulation have been carried out in rats and non-human primates. Up to the dose level tested of $450 \mathrm{mg} / \mathrm{kg}$ in rats ${ }^{22}$ and $750 \mathrm{mg} / \mathrm{kg}$ in non-human primates ${ }^{18}{ }^{26}$ with the Good Laboratory Pratice (GLP) or Good Manufacturing Practice (GMP) production batches, no obvious adverse effects were observed. After autopsy, the pathology showed only minimal and reversible renal tubular vacuolisation in rats due to the physiological elimination of the particles. Similar effect has been previously described for macrocyclic compounds such as cyclodextrin or derivatives, ${ }^{28}$ as well as gadolinium contrast agent like gadoteric acid. ${ }^{29}$ This vacuolisation only reported in rats is reversible and does not alter their renal function, even at repeated high doses. $^{25}$

The favourable profile of toxicity in animals, the straightforward intravenous administration, the imaging contrast agent and radiosensitising properties of AGuIX have motivated its use in patients as introduced by this first study in humans.

The medical cases for this study are patients with multiple brain metastases because of the technical difficulty of increasing the irradiation doses for all lesions without damaging the surrounding brain tissue and also because of the very poor prognosis of these patients.

\section{METHODS AND ANALYSIS \\ Study design}

This trial is a first in human, monocentric, open-label, dose-escalation phase I study to investigate the safety, tolerance and side effects' profile of AGuIX in combination with WBRT for patient with multiple brain metastases.

\section{Outcomes and assessments}

The primary outcome is the determination of the maximum-tolerated dose (MTD) of AGuIX given concurrently to the WBRT for the treatment of multiple brain metastases. The main criterion is determined by the incidence

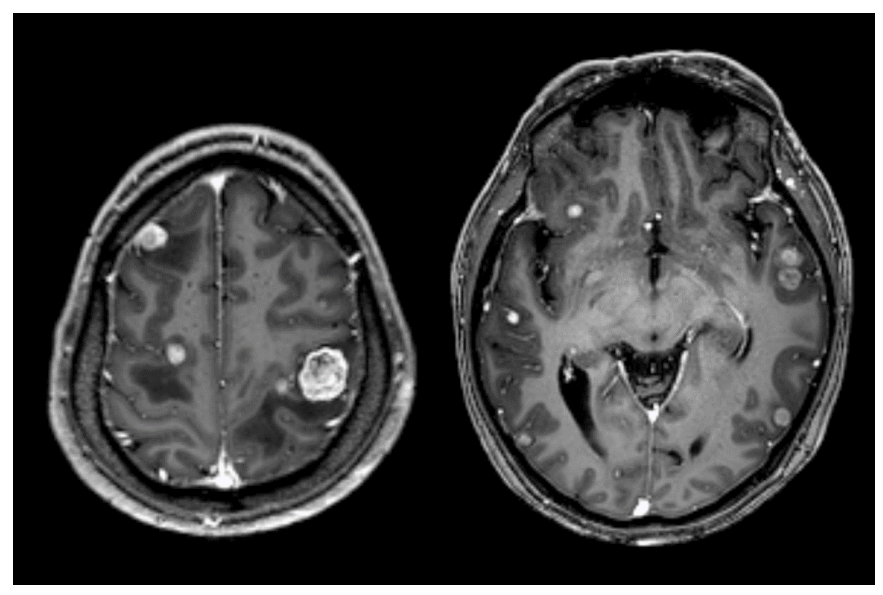

Figure 2 Illustration of multiple brain metastases on $\mathrm{T}_{1}$ injected MRI. The images correspond to inclusion criteria of NANO-RAD trial.

of dose limiting toxicity (DLT) graded using the NCI Common Terminology Criteria for Adverse Events V.4.03.

Secondary outcomes are:

- Pharmacokinetics profile of AGuIX particles, after intravenous administration, studied by quantification of gadolinium in blood and urinary sampling.

- Distribution of AGuIX particles in brain metastases and surrounding healthy tissue studied by MRI.

- Intracranial progression-free survival assessed by MRI (at 1, 3, 6, 9 and 12 months).

- Overall survival assessed from the date of discovery of the metastases.

\section{Patient selection}

Patients are recruited by experienced oncologists of the Radiation Oncology Department at the Grenoble Alpes University Hospital, according to the inclusion criteria. The decision of WBRT, including the systemic treatment strategy, should be previously validated by a multidisciplinary tumour board.

\section{Inclusion criteria}

- Patients with brain metastasis ineligible for local treatment by surgery or stereotactic irradiation (figure 2).

- At least 18 years old.

- Secondary brain metastases from a pathologically proven solid tumour.

- Patient able to give a written informed consent.

- Eastern Cooperative Oncology Group performance status $\leq 3$.

- No prior brain irradiation.

- No renal failure (glomerular filtration rate $>60 \mathrm{~mL} /$ $\left.\min / 1.73 \mathrm{~m}^{2}\right)$.

- Normal liver function (bilirubin $<30 \mu \mathrm{mol} / \mathrm{L}$; alkaline phosphatase $<400 \mathrm{UI} / \mathrm{L}$; aspartate aminotransferase $<75 \mathrm{UI} / \mathrm{L}$; alanine aminotransferase $<175 \mathrm{UI} / \mathrm{L}$ ).

- Affiliated to a social security insurance or assimilated.

\section{Exclusion criteria}

- Meningeal carcinomatosis. 
- Threatening extracranial disease.

- Previous brain irradiation (whether whole or partial brain radiation therapy, except stereotactic irradiation) or total body irradiation.

- Contra-indication, sensitivity or allergy to gadolinium.

- Patient unable to undergo or tolerate MRI.

- Patient participating to another clinical investigation at the same time.

- Person under juridical protection.

\section{Drug supply}

The gadolinium-based nanoparticles (AGuIX) are provided by NH TherAguix, Lyon, France, and are stored by the University Hospital Pharmacy, Grenoble.

\section{Interventions}

Patients are hospitalised in a phase I dedicated unit for 24 hours. On day 1 , patients receive a single intravenous injection of AGuIX (concentration $100 \mathrm{mg} / \mathrm{mL}$, rate flow $1 \mathrm{~mL} / \mathrm{min}$ ). Five dose escalation levels are planned: 15 , $30,50,75$ and $100 \mathrm{mg} / \mathrm{kg}$ in accordance with the retained dose escalation method, based on the modified Fibonacci sequence. An MRI is performed 2 hours after injection to visualise the distribution of AGuIX in brain metastases and surrounding healthy tissue, and to evaluate the contrast enhancement in brain metastases. Then, patients are treated by WBRT, starting 4 hours after AGuIX injection. During 24 hours after injection, several blood draws and urinary samples are collected in order to assess the pharmacokinetics of AGuIX. After completion of study treatment, patients' follow-up are done at D2, D8, M1, M3, M6, M9 and M12.

\section{Radiotherapy}

All patients are treated using the same protocol of WBRT with conventional linear accelerator. A personalised immobilisation device is made just before performing the dosimetric CT scan. The clinical target volume includes the whole brain with cerebellum and brainstem. Prescription dose is $30 \mathrm{~Gy}$ in 10 fractions of $3 \mathrm{~Gy}$; V95\%>95\%, Dmax $107 \%$. Patients will be treated by two opposite $6 \mathrm{MV}$ photon beams. Daily repositioning will be performed using ExacTrac system.

\section{Magnetic resonance imaging}

The MRI protocol is carried out on a 3T Achieva MR scanner (Philips Healthcare, Netherlands), at IRMaGE MRI facility (Grenoble, France).

MRI acquisitions cover the entire brain and consist of:

- A three-dimensional $\mathrm{T}_{1}$-weighted sequence, performed before and after DOTAREM or after AGuIX injection.

- $\mathrm{A} \mathrm{T}_{2}$ fluid attenuated inversion recovery sequence.

- A diffusion-weighted spin-echo sequence.

- A three-dimensional high-spatial resolution susceptibility weighted sequence.

- A $\mathrm{T}_{1}$-weighted fast-field-echo sequence. Four acquisitions will be performed using different flip angles $\left(5,15,20\right.$ and $\left.35^{\circ}\right)$ in order to calculate $\mathrm{T}_{1}$-maps used to estimate the gadolinium concentration in brain metastases.

This MRI protocol will be applied before the start of AGuIX combined with WBRT treatment (inclusion MRI), and on study days 1, 8 and 28. At inclusion and at day 28, an injection of DOTAREM is performed. At day 1, the sequences are carried out 2 hours after AGuIX injection.

\section{Pharmacokinetics analysis}

Blood samples are collected predose and at 0.25, 0.5, 1, 2, 4, 6, 10 and 24 hours postdose on study day 1 and on study day 8 , using sodium heparin collection tubes for trace elements (Becton Dickinson reference 367735, Pont de Claix, France). After centrifugation for $10 \mathrm{~min}$ at 2000x g (at room temperature), the plasma is collected, frozen and stored at $-20^{\circ} \mathrm{C}$ until analysis. A 24 hours fractional collection of urines is performed (3 fractions of 4 hours and 1 fraction of 12 hours). For each fraction, the diuresis is homogenised and two aliquots are removed, acidified (with ultrapure Normatom nitric acid) and stored at $-20^{\circ} \mathrm{C}$ until analysis.

Plasma and urinary concentrations of gadolinium are analysed using a validated inductively coupled plasma-mass spectrometry (ICP-MS) method. Briefly, gadolinium determination is performed by quadrupole ICP-MS equipped with quartz impact bead spray chamber, concentric nebuliser and Xt interface (X Serie II, Thermo Electron, Bremen, Germany) after dilution in nitric acid $1 \%(\mathrm{v} / \mathrm{V})$ containing $46 \mathrm{nmol}$ rhodium $(\mathrm{Rh})$ as internal standards. ${ }^{103} \mathrm{Rh},{ }^{155} \mathrm{Gd}$ and ${ }^{157} \mathrm{Gd}$ isotopes are measured against an external calibration curves. Plasma and urine spike with a known addition of Gd serve as internal quality control. For each patient, the dosages are carried out in the same series in order to limit the analytical variations. The analysis of dosages will provide the plasma half-life, the maximum plasma concentration, the area under the concentration-versus-time-curve (AUC) from time 0 to 24 hours after injection, the apparent distribution volume $(\mathrm{Vd})$ and the plasma clearance.

\section{Adverse events}

Toxicity is assessed using the NCI Common Toxicity Criteria V.4.03. DLT is defined for this study as the occurrence of grade 3 toxicity persistent 15 days after the end of radiotherapy or any grade 4 toxicity related to AGuIX injection. Adverse event will be systematically collected at each medical visit (D1, D2, D8, M1, M3, M6, M9 and M12) or during undercurrent events throughout the duration of the study. The accountability of the AGuIX will be established according to the time of occurrence and the type of symptoms. All the adverse events are transmitted to the Pharmacovigilance Unit and the analysis is carried out by an independent monitoring committee.

\section{Dose escalation design and MTD determination}

Patients will be enrolled in cohorts and will be treated at sequentially rising dose levels of AGuIX combined with WBRT. Three subjects will be initially recruited at each 
dose level. If none of these three patients experience a DLT, it will be proceed to the next dose level. If one of the three patients experiences toxicity, three more subjects will be recruited at that dose level. If at any time there are two or more dose-limiting toxicities (respectively in the three or six subjects) at a given dose level, the dose escalation will be stopped. Dose escalation will continue until the MTD of AGuIX and WBRT is established. The MTD will be defined as one dose level below the DLT occurring in at least one out of three subjects (two out of six patients).

A maximum of 18 patients will be included to achieve the study objectives.

\section{Statistical analysis}

The statistic threshold $(\alpha)$ held to consider a difference as statistically significant when $\mathrm{p} \leq 0.05$.

The Shapiro-Wilks test will be used to demonstrate the normality of the parameters. The Levene test will be used to demonstrate the homogeneity of the variances. When the conditions for applying the parametric tests are not satisfied, non-parametric tests can be performed.

The quantitative parameters for which normality will be admitted will be described by the mean $\pm \mathrm{SD}$, the $95 \%$ CI. They will be expressed as median, and the 25th and 75 th percentiles when normality will have been rejected. The qualitative parameters will be expressed in terms of numbers and percentages.

Statistical analyses will be carried out using the STATA software V.13 or higher (StataCorp).

\section{Follow-up}

Patients are followed during the first 24 hours hospitalisation, then at 8 days, 1, 3, 6, 9 and 12 months. Each visit includes a general examination, a neurological examination, a mini mental status assessment, an encephalic MRI and blood tests (blood cell count, electrolyte profile, liver enzymes, evaluation of renal function).

\section{Trial organisation and co-ordination}

The NANO-RAD study has been designed by the study investigators at the department of Radiation Oncology and the Clinical Research Department of Grenoble Alpes University Hospital. This study has been conceived in collaboration with NH TherAguix which develops the gadolinium-based nanoparticles AGuIX. The overall coordination is performed by the department of Radiation Oncology and the Clinical Investigation Center.

The Grenoble Alpes University Hospital is the sponsor of the study and is responsible for quality assurance, monitoring, reporting and database management. The study investigators are radiation oncologists, neuroradiologists, biologists specialised in brain metastases and dermatologists specialised in malignant melanoma.

All the results concerning the patients will be collected in an individual and anonymous case report form (CRF). The data will be entered in the CRF by the investigator and ascertained by the clinical research associate of the study. The data will be analysed by the biostatistician of the Clinical Research Department.

An independent review committee will review the data to assess the safety of the study. This committee will be solicited to validate each change of dose level.

\section{Ethics, informed consent and safety}

The study is conducted in accordance with the Declaration of Helsinki and with the recommendations of good clinical practice (ICHE6). The first patient was enrolled on 4 July 2016.

The data recorded in the context of this study are subject to a computerised treatment by the sponsor in compliance with the French law $n{ }^{\circ} 78-17$ of the 6 January 1978 relating to data processing, files and freedoms, modified by the law 2004-801 of the 6 August 2004 .

Each patient recruited into the study, has to provide his written informed consent prior to inclusion after extensive information about the intent of the study and potential side effect. The written informed consents are collected by the main investigator of the trial. Participant confidentiality will be ensured and anonymity guaranteed.

\section{Dissemination}

The analysis of the primary and secondary endpoints will be performed immediately after completion of data collection which is expected for 2019. We aim to disseminate the results by publication in a peer-reviewed and to present the study at national and international oncology meetings.

\section{Patient and public involvement}

Patient and public were not involved in the design and conduction of the study.

\section{DISCUSSION}

The lack of progress for decades in the management of patients with multiple brain metastases is due to three factors: the multiplicity of brain lesions, their radioresistance comparing to the brain and the poor distribution of cytotoxic agents in brain metastases. Therefore, the use of new radiosensitisers is worth to be explored in the treatment of multiple brain metastases. The objective is to increase the dose delivered within the metastases to improve local control of metastases and overall survival without increasing the dose in the surrounding healthy tissues. Preclinical studies suggest that AGuIX gadolinium nanoparticles, after a single intravenous injection, are able to enhance the image of all metastases and to increase the efficiency of the irradiation. ${ }^{13182022}$

The goal of this study is to perform the first in human injection, to evaluate the safety and to determine the MTD of AGuIX combined with WBRT for treatment of multiple brain metastases.

Author affiliations

'Department of Radiotherapy, Grenoble Alpes University Hospital, Grenoble, France 
${ }^{2}$ Grenoble Institute of Neurosciences, INSERM U1216, Grenoble Alpes University, Grenoble, France

${ }^{3}$ Institute Light and Mater, UMR5306, Lyon1 University-CNRS, Lyon University, Villeurbanne, France

${ }^{4} \mathrm{NH}$ TherAguix, NH TherAguix SAS, Villeurbanne, France

${ }^{5}$ Clinical Investigation Center, Grenoble Alpes University Hospital, Grenoble, France

${ }^{6}$ Department of Neuroradiology, Grenoble Alpes University Hospital, Grenoble, France

${ }^{7}$ Institute of Biology and Pathology, Unit of Hormonal and Nutritional Biochemistry, Grenoble Alpes University Hospital, Grenoble, France

Acknowledgements The authors gratefully acknowledge the IRMaGe MRI facility, that is partly funded by the French programme Investissement d'Avenir" run by the "Agence Nationale pour la Recherche"; grant Infrastructure d'avenir en Biologie Santé" - ANR-11-INBS-0006" and by France Life Imaging.

Contributors CV, JB, LS, OT andJLQ designed the study. CV, LS, JA andJLQ wrote the protocol. CV is the trial co-ordinator and the main investigator. CV, JV andJB participate in the patient recruitment. CM participates in trial coordination. SG participates in MRI analysis. JA participates in Gd analysis in biological samples. JLQ supervises statistical analysis. CV and SD wrote the paper and GLD, FL, LS, $J A, J L Q$ andJB revised it critically. All authors contributed to and approved the final version of the manuscript.

Funding The trial is partially funded by NH TherAguix (Villeurbanne, France.). NH TherAguix has reviewed the design of the study, and is involved in analysis and interpretation of the data, and in writing the manuscript.

Competing interests FL and OT have to disclose the patent W02011/135101. GLD and OT have to disclose the patent W02009/053644. These patents protect the AGuIX® nanoparticles described in this publication. SD and GLD are employees from NH TherAguix that is developing the AGuIX® nanoparticles. The trial medication (AGuIX®) and a financial grant for study organization are supplied by NH TherAguix, Villeurbanne, France.

Patient consent Obtained.

Provenance and peer review Not commissioned; externally peer reviewed.

Open access This is an open access article distributed in accordance with the Creative Commons Attribution Non Commercial (CC BY-NC 4.0) license, which permits others to distribute, remix, adapt, build upon this work non-commercially, and license their derivative works on different terms, provided the original work is properly cited, appropriate credit is given, any changes made indicated, and the use is non-commercial. See: http://creativecommons.org/licenses/by-nc/4.0/.

\section{REFERENCES}

1. Scoccianti S, Ricardi U. Treatment of brain metastases: review of phase III randomized controlled trials. Radiother Oncol 2012;102:168-79.

2. Murray KJ, Scott C, Greenberg HM, et al. A randomized phase III study of accelerated hyperfractionation versus standard in patients with unresected brain metastases: a report of the Radiation Therapy Oncology Group (RTOG) 9104. Int J Radiat Oncol Biol Phys 1997;39:571-4.

3. Mehta MP, Rodrigus $\mathrm{P}$, Terhaard $\mathrm{CH}$, et al. Survival and neurologic outcomes in a randomized trial of motexafin gadolinium and whole-brain radiation therapy in brain metastases. J Clin Oncol 2003;21:2529-36.

4. Posner JB. Brain metastases: 1995. A brief review. J Neurooncol 1996;27:287-93.

5. Patchell RA, Tibbs PA, Regine WF, et al. Postoperative radiotherapy in the treatment of single metastases to the brain: a randomized trial. JAMA 1998;280:1485-9.

6. Chua D, Krzakowski M, Chouaid C, et al. Whole-brain radiation therapy plus concomitant temozolomide for the treatment of brain metastases from non-small-cell lung cancer: a randomized, openlabel phase II study. Clin Lung Cancer 2010;11:176-81.

7. Shaw E, Scott C, Suh J, et al. RSR13 plus cranial radiation therapy in patients with brain metastases: comparison with the radiation therapy oncology group recursive partitioning analysis brain metastases database. J Clin Oncol 2003;21:2364-71.

8. Suh JH, Stea B, Nabid A, et al. Phase III study of efaproxiral as an adjunct to whole-brain radiation therapy for brain metastases. $J$ Clin Oncol 2006;24:106-14.

9. Bradley KA, Pollack IF, Reid JM, et al. Motexafin gadolinium and involved field radiation therapy for intrinsic pontine glioma of childhood: a Children's Oncology Group phase I study. Neuro Oncol 2008;10:752-8.

10. Hainfeld JF, Slatkin DN, Smilowitz HM. The use of gold nanoparticles to enhance radiotherapy in mice. Phys Med Biol 2004:49:N309-15.

11. Maggiorella L, Barouch G, Devaux C, et al. Nanoscale radiotherapy with hafnium oxide nanoparticles. Future Oncol 2012;8:1167-81.

12. Lux F, Mignot A, Mowat $P$, et al. Ultrasmall rigid particles as multimodal probes for medical applications. Angew Chem Int Ed Engl 2011;50:12299-303.

13. Kotb S, Detappe A, Lux F, et al. Gadolinium-based nanoparticles and radiation therapy for multiple brain melanoma metastases: proof of concept before phase I trial. Theranostics 2016:6:418-27.

14. Luchette $\mathrm{M}$, Korideck $\mathrm{H}$, Makrigiorgos $\mathrm{M}$, et al. Radiation dose enhancement of gadolinium-based AGuIX nanoparticles on HeLa cells. Nanomedicine 2014:10:1751-5.

15. Mowat $P$, Mignot $A$, Rima W, et al. In vitro radiosensitizing effects of ultrasmall gadolinium based particles on tumour cells. J Nanosci Nanotechnol 2011:11:7833-9.

16. Porcel E, Tillement O, Lux F, et al. Gadolinium-based nanoparticles to improve the hadrontherapy performances. Nanomedicine 2014;10:1601-8.

17. Sancey L, Lux F, Kotb S, et al. The use of theranostic gadoliniumbased nanoprobes to improve radiotherapy efficacy. Br J Radiol 2014;87:20

18. Detappe A, Kunjachan S, Sancey L, et al. Advanced multimodal nanoparticles delay tumor progression with clinical radiation therapy. $J$ Control Release 2016;238:103-13.

19. Dufort S, Bianchi A, Henry M, et al. Nebulized gadolinium-based nanoparticles: a theranostic approach for lung tumor imaging and radiosensitization. Small 2015;11:215-21.

20. Le Duc G, Miladi I, Alric C, et al. Toward an image-guided microbeam radiation therapy using gadolinium-based nanoparticles. ACS Nano 2011:5:9566-74.

21. Miladi I, Aloy MT, Armandy E, et al. Combining ultrasmall gadolinium-based nanoparticles with photon irradiation overcomes radioresistance of head and neck squamous cell carcinoma. Nanomedicine 2015;11:247-57.

22. Verry C, Dufort S, Barbier EL, et al. MRI-guided clinical 6-MV radiosensitization of glioma using a unique gadolinium-based nanoparticles injection. Nanomedicine 2016;11:2405-17.

23. Lux F, Sancey L, Bianchi A, et al. Gadolinium-based nanoparticles for theranostic MRI-radiosensitization. Nanomedicine 2015;10:1801-15.

24. Maeda H, Sawa T, Konno T. Mechanism of tumor-targeted delivery of macromolecular drugs, including the EPR effect in solid tumor and clinical overview of the prototype polymeric drug SMANCS. J Control Release 2001;74(1-3):47-61.

25. Sancey L, Kotb S, Truillet C, et al. Long-term in vivo clearance of gadolinium-based AGuIX nanoparticles and their biocompatibility after systemic injection. ACS Nano 2015:9:2477-88.

26. Kotb S, Piraquive J, Lamberton F, et al. Safety evaluation and imaging properties of gadolinium-based nanoparticles in nonhuman primates. Sci Rep 2016;6:35053.

27. Bianchi A, Dufort S, Lux F, et al. Targeting and in vivo imaging of nonsmall-cell lung cancer using nebulized multimodal contrast agents. Proc Natl Acad Sci U S A 2014;111:9247-52.

28. Bostan H, Kalkan Y, Tomak Y, et al. Reversal of rocuroniuminduced neuromuscular block with sugammadex and resulting histopathological effects in rat kidneys. Ren Fail 2011;33:1019-24.

29. DOTAREM. Guerbet: Résumé des caractéristiques du produit. 2013. 